\title{
On modeling two-mode vibration sensors for combined density and viscosity measurements
}

\author{
T. Wattinger, L. Zartmann \& J. Dual \\ Institute of Mechanical Systems, Department of Mechanical and \\ Process Engineering, ETH Zurich, Switzerland
}

\begin{abstract}
Torsional and bending mode vibrations of a hollow cylinder shall be used for combined measurement of density and viscosity of a fluid. The employment of two different vibration modes has the advantage that modes with good sensitivity for either density or viscosity measurements can be used. For a detailed discussion of the dependencies of the sensitivity, a description of the fluid forces and a model of the sensor are required. Analytical solutions for the fluid forces acting on an oscillating cylindrical structure usually consider a two-dimensional situation. FEM simulations are performed in order to investigate the suitability of such solutions in order to describe fluid forces in a three-dimensional situation and for different bending modes. For the case at hand, an analytical relationship between the resonance frequency and the density of the liquid can be formulated. This analytical relationship forms a valuable basis for further investigations.

Keywords: vibration sensor, bending, density and viscosity.
\end{abstract}

\section{Introduction}

In many industrial production processes the control of fluid properties plays a key role. Monitoring of fluid density and viscosity is of particular importance in food, cosmetics, petrochemical, and general chemical industry. Examples of applications are density and concentration measurements in beverages, in intermediate products of refineries or in fuel blends.

In the literature several resonator sensors for the combined measurement of density and viscosity are proposed. Krall and Sengers [1] evaluates the damping and oscillation period of an oscillating disk. Da Mata et al. [2] presents a vibratingwire sensor, for which the tension in the wire is determined by the hydrostatic 
forces on a sinker, that is attached to one end of the wire. The sensor is run in forced mode, from which resonance frequency and bandwidth of the resonance peak are extracted, and in free decay mode, from which oscillation frequency and logarithmic decrement are obtained. From these quantities the fluid properties can be determined.

A detailed theory and design criteria for density and viscosity sensors based on flexural vibrations of a rod which is clamped on both ends and surrounded by a fluid are presented in Retsina et al. [3, 4]. Here too, the use of two different modes of operation is proposed. A forced mode where the resonance frequency is measured in order to obtain the density of the liquid (viscosity known). Then the setup is run in free decay mode, where the logarithmic decrement is measured in order to obtain the viscosity of the fluid (density known). It must be noted, that both density and viscosity could be measured solely with one mode of operation (forced or free decay), but either the viscosity or density obtained this way would be less accurate. An application of the presented theory is given in Bett et al. [5].

In the patent literature (Fitzgerald [6]), an in-line vibratory viscometer densitometer is found which consists of a hollow tube clamped on both ends, and with the fluid flowing through the tube. The tube is excited to simultaneously oscillate in a flexural and a torsional mode. The power required to maintain a constant amplitude of the torsional vibrations is related to the fluid viscosity. The fluid density can be inferred from the resonance frequency of the flexural vibrations.

Literature on combined density and viscosity measurement is also found for micromechanical sensors. Examples are vibrating plates (Goodwin et al. [7]) or vibrating microcantilevers (Ghatkesar et al. [8]). The setup in Ghatkesar et al. [8] consists of arrays of prismatic microcantilevers. The presented experimental study investigates the amplitude and phase spectra of these cantilevers for 16 different modes, and in different fluids. Some interesting observations considering the sensitivity of different modes on density and viscosity are presented.

The sensors discussed above, except those of Fitzgerald [6] and Ghatkesar et al. [8], make use of only one oscillation mode (usually the fundamental mode). As a consequence thereof, usually only one fluid property (density or viscosity) can be determined with satisfying accuracy. These measurements therefore rely on the knowledge of one fluid property from the literature, from other measurements or have to employ another vibrational mode. Clearly, the employment of different oscillation modes with good sensitivity for either density or viscosity would be beneficial.

The aforementioned sensors rely on a theoretical model to relate measured quantities to the fluid properties. These models in turn require a proper description of the fluid forces acting on the oscillating structures. An aspect which seems to have gained little interest so far are edge effects in the description of the fluid forces. In Retsina et al. [3] for example, it is assumed that the radius of the oscillating rod is much smaller than the length of the rod, and axial effects can be neglected. Also the theoretical model to which the measurements in Ghatkesar et al. [8] are compared, is based on fluid forces calculated for a thin blade of infinite 
length. A more detailed investigation of such boundary effects would provide a better basis to judge their influence on the measurements.

In the present work a sensor is considered which is based on a hollow cylinder that performs torsional and flexural oscillations. The employment of these different vibration modes has the advantage that good sensitivity can be achieved for either density or viscosity measurements. In this regard the functionality of an existing viscosity sensor (Dual [9], Dual and O'Reilly [10] and Hausler et al. [11]) based on a torsionally vibrating rod shall be extended to include the ability to perform density measurements using bending vibrations.

In this text, the modeling of a flexurally vibrating hollow cylinder for density measurements is considered. The setup and the measurement principle of the sensor are presented in section 2. In section 3.1 a mechanical model of the resonator is introduced. Section 3.2 is owed to the description of the fluid forces. Finally in section 3.3 an analytical model of the fluid-loaded sensor is presented.

\section{Setup and measurement principle}

The sensor which is used in this work is sketched in Fig. 1. It consists of a body $(a)$, an outer hollow cylinder $(b)$ which on his outer face is in contact with the fluid, a connecting piece $(c)$ which makes a connection to an inner hollow cylinder $(d)$ where at the free end a permanent magnet $(e)$ is attached. A coil bobbin with two coils $(f)$ is mounted on the body. The coils and the polarization of the permanent magnet are aligned in a way such that an alternating voltage leads to bending or torsion moments acting on the permanent magnet. Therefore the sensor can be excited to perform either flexural or torsional vibrations. The sensor is in contact with the fluid along the outer surface of the outer hollow cylinder and the front side of the connecting piece.

A phase-locked loop (PLL) is used in order to measure the resonance frequency and damping of the oscillating system. The PLL allows to stabilize the phase between the harmonic excitation signal and the harmonic velocity of the oscillating structure. Under laboratory conditions, the velocity of a point on the outside of the

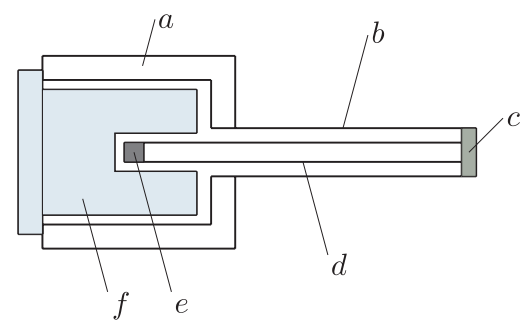

Figure 1: Schematic representation of the resonator sensor (arbitrarily scaled). $(a)$ sensor body, $(b)$ outer hollow cylinder, $(c)$ connecting piece, $(d)$ inner hollow cylinder, $(e)$ permanent magnet, $(f)$ coil bobbin with coils. 
outer cylinder can be measured using a laser doppler vibrometer. Otherwise the induction caused by the permanent magnet in a measuring coil is evaluated to measure the velocity.

The PLL controls the excitation frequency in order to maintain the desired phase between excitation and velocity signal. If excitation moment and velocity are in phase (phase difference of $0^{\circ}$ ) the system is in resonance.

\section{Modeling}

This chapter discusses different aspects of a model describing the flexural vibrations of the sensor. A detailed model regarding torsional vibrations can be found in Dual [9].

\subsection{Description of the mechanical resonator}

The outer hollow cylinder of the sensor which is in contact with the surrounding fluid forms the actual 'sensing' part. The inner hollow cylinder, while being part of the oscillating structure, is basically necessary to excite the structure. In a first modeling approach the sensor can be described as a beam which is clamped at one end and excited by a moment acting on the free end. The situation under consideration is sketched in Fig. 2. The beam dimensions and material parameters are given by the outer hollow cylinder of the sensor. They are given as outer radius $R$, beam length $L$, cross-sectional area $A$, second moment of area $I_{z}$, density $\rho_{B}$ and Young's modulus $E$. The harmonic excitation is described by the moment $M(t)=M_{0} e^{i \omega t}$.

The above consideration fairly simplifies the actual situation. A more detailed description is desirable but it turns out that it is extremely difficult to build an analytical model that accurately describes the complete sensor assembly. The proposed simple model nevertheless provides some useful insights into the fluidstructure interaction of the sensor.

Applying Euler-Bernoulli beam theory, the differential equation describing the deflection $u(x, t)$ of the flexural vibrations is given by eqn. (1) in which $q(x, t)$ is a distributed transverse force.

$$
\rho_{B} A u_{, t t}+E I_{z} u_{, x x x x}=q(x, t)
$$

The solution of the above equation is of the form

$$
\begin{gathered}
u(x, t)=\left[\tilde{a} e^{i k x}+\tilde{b} e^{-i k x}+\tilde{c} e^{k x}+\tilde{d} e^{-k x}\right] e^{i \omega t} \\
\mathbb{y} \int^{\mathrm{y}(\mathrm{t})}
\end{gathered}
$$

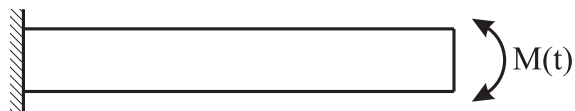

Figure 2: Simplified beam model. 
in which $k$ is the wavenumber and $\tilde{a}, \tilde{b}, \tilde{c}, \tilde{d}$ are complex constants that are determined by the boundary conditions. In vacuum the wavenumber is given as $k_{0}^{4}=\left(\rho_{B} A \omega_{0}^{2}\right) /\left(E I_{z}\right)$ with $\omega_{0}$ as the angular rotational velocity in vacuum.

\subsection{Description of fluid forces}

The fluid considered in this work is a viscous, incompressible, Newtonian fluid.

In the case of a beam immersed in a fluid, the distributed transverse force $q(x, t)$ in eqn. (1) describes the influence of the surrounding fluid on the oscillating beam. A viscous fluid has two effects on the oscillations of the beam. It leads to a viscous damping force and to an additionally accelerated mass (added mass effect). The former describes a force proportional to the beam velocity while the latter is proportional to the beam acceleration. The distributed transverse force can therefore generally be expressed as

$$
q(x, t)=(\alpha+i \beta) u_{, t}
$$

in which $\alpha$ and $\beta$ describe damping force and added mass, respectively, and are frequency dependent.

Analytical solutions for $\alpha$ and $\beta$ in the case of harmonically oscillating cylinders in viscous, incompressible fluids can be found in literature (Kremlevskii and Stepichev [12], Retsina et al. [3]). A solution taking into account the effect of a cylindrical boundary around the fluid is presented in Chen et al. [13]. All of these analytical solutions consider a two-dimensional situation, i.e. a rigid, infinitely long cylinder. As proposed by Kremlevskii and Stepichev [12], one could think of the beam to be composed of infinitesimally thin disks, and for each of them the two-dimensional solution holds. The question arises, how accurate such a description reproduces the actual three-dimensional situation. Especially in the present case of a relatively short clamped-free beam, it is of interest how accurate the fluid forces can be described near the free end of the beam and how the force distribution changes depending on mode shapes. In order to answer these questions, the analytical results are contrasted with results from FEM simulations.

A FEM model incorporating the entire mechanical structure of the sensor, a description of the fluid, and the fluid-structure interaction would become very large and cannot be handled computationally. In order to investigate the fluid forces acting on the flexurally oscillating outer hollow cylinder of the sensor, it is for most cases sufficient to consider a cylindrical wall which oscillates according to the deflection pattern under vacuum. (This implies that the mode shapes do not change under the influence of the fluid.) For the simulation considered here, the deflection pattern is extracted from an eigenfrequency analysis of a detailed FEM model of the entire sensor (Fig. 1). The fluid domain is bounded by a cylindrical wall with radius $R_{b}$. Simulations are carried out using the COMSOL Multiphysics software. The fluid is modelled as an incompressible, viscous fluid and is described by the Navier-Stokes equations. All parameters relevant for the simulation are summarized in Table 1. (The ratio of the radii $R_{b} / R$ is 8 . According to Chen et al. [13] the influence of the fluid boundary becomes negligible in this case.) 
Table 1: Simulation parameters.

\begin{tabular}{|l|c|}
\hline Cylinder radius $R$ & $0.004 \mathrm{~m}$ \\
\hline Cylinder length $L$ & $0.0605 \mathrm{~m}$ \\
\hline Boundary radius $R_{b}$ & $0.032 \mathrm{~m}$ \\
\hline Fluid density $\rho$ & $844 \mathrm{~kg} / \mathrm{m}^{3}$ \\
\hline Fluid viscosity $\eta$ & $978 \mathrm{mPa} s$ \\
\hline
\end{tabular}

For the evaluation of the simulation the oscillating cylindrical wall is divided into small disks and the fluid force (component in the direction of the oscillation) is integrated over the surface of each disk. Also the average amplitude of the deflection velocity for each disk is extracted. Applying the description for the fluid forces according to Chen et al. [13], the extracted velocities can be used to calculate the theoretical fluid forces. These can then be contrasted to the integrated forces. The results (for second and third bending modes) are shown in Fig. 3, where the magnitude of the fluid forces along the cylinder is displayed.

Both graphs in Fig. 3 show a good correspondence of the fluid forces from simulation and analytical description except near the free end of the cylinder. At the free end, the force obtained from FEM simulation is smaller than the analytically calculated force. This can be explained by the fluid flowing around the free end of the beam instead of flowing in a plane perpendicular to the beam axis. Due to this shunting of the fluid, the theoretically expected fluid forces cannot build up. As the region with larger deviations between the fluid forces according to simulation and analytical description pertain to a small part of the whole cylinder surface, its influence on the oscillation is not expected to be dramatic. At least for the considered case the analytical description seems to be appropriate to describe the fluid forces in a three-dimensional case.
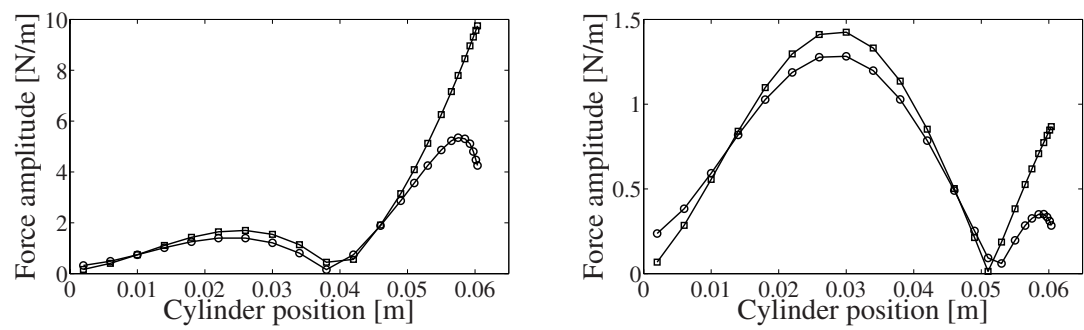

Figure 3: Distribution of fluid forces along the flexurally oscillating outer cylindrical surface of the sensor according to FEM simulation (o) and according to an analytical description from Chen et al. [13] ( $\square$ ). Left: Second bending mode. Right: Third bending mode. 


\subsection{Analytical model for fluid-loaded resonator}

Although FEM is a valuable tool and offers solutions to model fluid-structure interaction problems, we strive here for an analytical model. The motivation is to obtain a model, which allows solving the inverse problem, i.e. to relate resonance frequency to fluid density. Such a model then allows performing parameter studies in order to find an optimal sensor design. Further, in contrast to a phenomenologically based relation between measured quantities and fluid properties, an analytical model can provide useful insight into the physics of the problem.

In this section an analytical model for a cylindrical beam which is clamped at one end and excited by a moment acting on the free end (Fig. 2) and which is surrounded by a viscous incompressible fluid is derived. The fluid domain is considered as unbounded.

In this model the fluid forces are expressed according to Kremlevskii and Stepichev [12] for the case that the penetration depth $\delta=\sqrt{\left(\frac{2 \eta}{\rho \omega}\right)}$ (in which $\rho$ and $\eta$ denote fluid density and viscosity respectively) in the fluid is much smaller than the radius $R$ of the cylinder $(\delta / R \ll 1)$. The factors $\alpha$ and $\beta$ in the distributed transverse force $q(x, t)$ (eqn. (3)) can therefore be written as

$$
\alpha=-2 \sqrt{2} \pi R \sqrt{\omega \rho \eta}, \quad \beta=-2 \sqrt{2} \pi R \sqrt{\omega \rho \eta}-\pi R^{2} \rho \omega
$$

Applying the separation ansatz $u(x, t)=e^{k x} e^{i \omega t}$ leads to the following equation for the wavenumber $k$ :

$$
\frac{k^{4}}{k_{0}^{4}}=1-\frac{\beta}{\rho_{B} A \omega}+i \frac{\alpha}{\rho_{B} A \omega}=P e^{i \varphi}
$$

in which $P, \varphi$ are magnitude and phase of the ratio $k^{4} / k_{0}^{4}$ and are introduced for convenience.

The wavenumber $k$ is complex, and will be denoted as

$$
k_{c}=k_{0} P^{1 / 4}[\cos (\varphi / 4)+i \sin (\varphi / 4)]=k_{r}+i k_{i}
$$

Employing the complex wavenumber $k_{c}$ in eqn. (1) and taking into account the boundary conditions according to Fig. 2, a transfer function relating the angular rotational velocity $w=u_{, x t}$ to the amplitude $M_{0}$ of the moment excitation can be derived:

$$
\frac{w}{M_{0}}=\frac{i \omega\left[\cosh \left(k_{c} L\right) \sin \left(k_{c} L\right)+\cos \left(k_{c} L\right) \sinh \left(k_{c} L\right)\right]}{E I_{z} k_{c}\left[1+\cos \left(k_{c} L\right) \cosh \left(k_{c} L\right)\right]}
$$

Resonances are found in above transfer function at oscillation states where the phase is zero or where the imaginary part of the transfer function is zero. These states are defined by the complex wavenumber times beam length product $k_{c} L$ or in more detail on the relation between $k_{r} L$ and $k_{i} L$. Fig. 4 shows a contour plot describing the ratios between $k_{r} L$ and $k_{i} L$ for which resonances occur. Numerical evaluations of the above transfer function shows that for densities between 400 


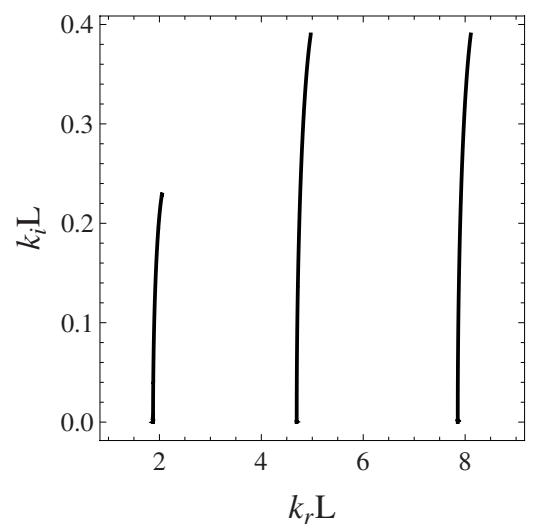

Figure 4: Contour plot of $k_{r} L$ and $k_{i} L$ at resonance.

and $2000 \mathrm{~kg} / \mathrm{m}^{3}$ and viscosities between 1 and $1000 \mathrm{mPa} s$ the values of $k_{i} L$ stays below 0.05 . In this region the values of $k_{r} L$ are almost constant. (The ratio between $k_{r} L\left(k_{i} L=0.05\right) / k_{r} L\left(k_{i} L=0\right)$ is 1.0029 for mode 1 and 1.0004 for mode 3 .)

Assuming therefore $k_{r}$ to be constant and $\varphi=0$ one can write $k_{r}=k_{0} P^{1 / 4}$. A series expansion of $P$ around an arbitrary value $\rho=\rho_{0}$ for the fluid density up to powers of $\left(\rho-\rho_{0}\right)^{1}$ and reformulating the expression for $k_{r}$ leads to the expression

$$
\rho=\rho_{0}\left(1+\frac{\left(\frac{\omega_{0}}{\omega}\right)^{2} \sqrt{(\quad)}-(\quad)}{\tilde{m}[\quad]}\right)
$$

in which

$$
(\quad)=8 \tilde{\delta}^{2} \tilde{m}^{2}+(\tilde{m}+1)(\tilde{m}+4 \tilde{\delta} \tilde{m}+1) \quad[\quad]=4 \tilde{\delta}^{2} \tilde{m}+(\tilde{m}+1)+\tilde{\delta}(3 \tilde{m}+1)
$$

and $\omega$ is the angular rotational velocity of the beam in the fluid, $\tilde{\delta}=\delta_{0} / R$ with $\delta_{0}=\sqrt{\left(\frac{2 \eta}{\rho_{0} \omega}\right)}$ as the penetration depth in a fluid with density $\rho_{0}$, and $\tilde{m}=\pi R^{2} \rho_{0} /\left(A \rho_{B}\right) . \tilde{m}$ is denoted as the mass ratio between fluid and sensor.

Having this formula at hand, it is now possible to relate the fluid density to the resonant frequency in fluid.

The quantities that need to be known in order to evaluate eqn. (8) are the angular rotational velocity in vacuum $\omega_{0}$, the outer radius $R$ of the cylindrical beam, fluid viscosity $\eta$, and the mass per unit length of the beam $A \rho_{B}$. The angular rotational velocity in vacuum $\omega_{0}$ and the outer radius $R$ can be measured. The fluid viscosity $\eta$ is obtained from the viscosity measurement. The mass per unit length of the beam $A \rho_{B}$ is best evaluated through calibration measurements, because both the material density $\rho_{B}$ and the cross-sectional area $A$ of the hollow cylinder are not easily determined accurately. 

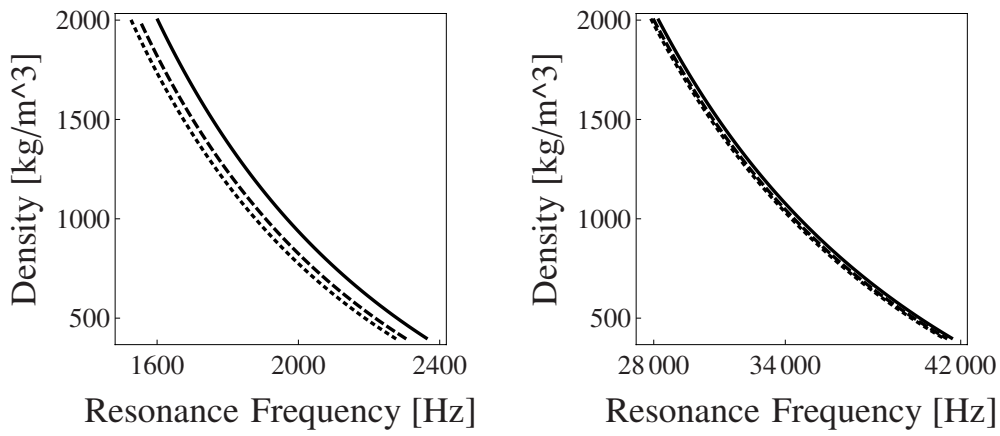

Resonance Frequency $[\mathrm{Hz}]$

Figure 5: Relation between fluid density $\rho$ and resonance frequency. Continuous, dashed, and dotted lines correspond to viscosities of 10, 500, $1000 \mathrm{mPa} s$. Left: First bending mode. Right: Third bending mode.

Fig. 5 shows the relation between fluid density $\rho$ and the resonance frequency for the simplified beam model and for the first and third bending mode.

After a first look at Fig. 5, it seems that the influence of viscosity on the resonance frequency at a certain fluid density is less pronounced for the third than for the first mode. However, this cannot be directly assessed because of the different frequency ranges of the two modes. Consider therefore the resonance frequencies at a density of $\rho_{1}=1000 \mathrm{~kg} / \mathrm{m}^{3}$ and for viscosities of $\eta_{1}=10 \mathrm{mPa} s$ and $\eta_{3}=1000 \mathrm{mPa} s$. The frequency difference $\Delta_{\text {fres }}=f_{\text {res }}\left(\rho_{1}, \eta_{1}\right)-$ $f_{\text {res }}\left(\rho_{1}, \eta_{3}\right)$ obtained for the first mode is $89 \mathrm{~Hz}$ and for the third mode $378 \mathrm{~Hz}$. The ratio between these frequency differences and the resonance frequency obtained for viscosity $\eta_{1}$, i.e. $\Delta_{\text {fres }} / f_{\text {res }}\left(\rho_{1}, \eta_{1}\right)$, takes the values 0.045 in the case of the first mode and 0.011 in the case of the third mode. At least for the considered case it can therefore be concluded, that the effect of viscosity on the density-resonance frequency relation is smaller for the third bending mode than for the first bending mode.

\section{Summary and conclusions}

A simplified beam model is investigated to gain insight into the fluid-structure interaction of a sensor. In order to build an analytical model of the sensor, a description of the fluid forces is necessary. FEM simulations show that an analytical description of the fluid forces in two dimensions is capable to describe the actually three-dimensional situation. An analytical relation between resonance frequency of the resonator in fluid and the fluid density is derived. This relation is a valuable basis for further investigations of the fluid-structure interaction. Parameter studies can now be performed to establish design criteria for optimal measurement sensitivity. 


\section{References}

[1] Krall, A.H. \& Sengers, J.V., Simultaneous measurement of viscosity and density with an oscillating-disk instrument: The effect of fixed plates. International Journal of Thermophysics, 24(2), pp. 337-359, 2003.

[2] da Mata, J. et al. A new instrument to perform simultaneous measurements of density and viscosity of fluids by a dual vibrating-wire technique. High Temperatures-High Pressures, 33(6), pp. 669-676, 2001.

[3] Retsina, T. et al. The theory of a vibrating-rod densimeter. Applied Scientific Research, 43(2), pp. 127-158, 1986.

[4] Retsina, T. et al. The theory of a vibrating-rod viscometer. Applied Scientific Research, 43(4), pp. 325-346, 1987.

[5] Bett, K.E. et al. A vibrating-rod densimeter. International Journal of Thermophysics, 10(4), pp. 871-883, 1989.

[6] Fitzgerald, J.V., In-line vibratory viscometer-densitometer. patent number: 4524610. United States Patent, 1985.

[7] Goodwin, A.R.H. et al. A mems vibrating edge supported plate for the simultaneous measurement of density and viscosity: Results for nitrogen, methylbenzene, water, 1-propene,1,1,2,3,3,3=hexafluoro-oxidized-polymd, and polydimethylsiloxane and four certified reference materials with viscosities in the range ( 0.038 to 275 ) mpa.s and densities between (408 and 1834) kg.m(-3) at temperature from (313 to 373 ) k and pressure up to $60 \mathrm{mpa}$. Journal of Chemical and Engineering Data, 53(7), pp. 1436-1443, 2008.

[8] Ghatkesar, M.K. et al. Multi-parameter microcantilever sensor for comprehensive characterization of newtonian fluids. Sensors and Actuators B-Chemical, 135(1), pp. 133-138, 2008.

[9] Dual, J., Experimental methods in wave propagation in solids and dynamic viscometry. Dissertation, doi:103929/ethz-a-000514793, 1989.

[10] Dual, J. \& O'Reilly, O.M., Resonant torsional vibrations - an application to dynamic viscometry. Archive of Applied Mechanics, 63(7), pp. 437-451, 1993.

[11] Hausler, K. et al. A newly designed oscillating viscometer for blood viscosity measurements. Biorheology, 33(4-5), pp. 397-404, 1996.

[12] Kremlevskii, V.P. \& Stepichev, A.A., Measurement of viscosity and density of a liquid by means of a vibratory transducer. Soviet Physics Acoustics-Ussr, 21(1), pp. 37-40, 1975.

[13] Chen, S.S. et al. Added mass and damping of a vibrating rod in confined viscous fluids. Journal of Applied Mechanics-Transactions of the ASME, 43(2), pp. 325-329, 1976. 